\title{
Breakup of loosely bound nuclei as indirect method in nuclear astrophysics: ${ }^{8} \mathrm{~B},{ }^{9} \mathrm{C},{ }^{23} \mathrm{Al}$
}

\author{
L. Trache ${ }^{1, a}$, F. Carstoiu ${ }^{2}$, C.A. Gagliardi ${ }^{1}$, and R.E. Tribble ${ }^{1}$ \\ 1 Cyclotron Institute, Texas A\&M University, College Station, TX 77843-3366, USA \\ 2 National Institute of Physics and Nuclear Engineering H. Hulubei, Bucharest, Romania
}

Received: 25 August 2005 /

Published online: 10 March 2006 - (C) Società Italiana di Fisica / Springer-Verlag 2006

\begin{abstract}
We discuss the use of one-nucleon breakup reactions of loosely bound nuclei at intermediate energies as an indirect method in nuclear astrophysics. These are peripheral processes, therefore we can extract asymptotic normalization coefficients (ANC) from which reaction rates of astrophysical interest can be inferred. To show the usefulness of the method, three different cases are discussed. In the first, existing experimental data for the breakup of ${ }^{8} \mathrm{~B}$ at energies from 30 to $1000 \mathrm{MeV} / u$ and of ${ }^{9} \mathrm{C}$ at $285 \mathrm{MeV} / u$ on light through heavy targets are analyzed. Glauber model calculations in the eikonal approximation and in the optical limit using different effective interactions give consistent, though slightly different results, showing the limits of the precision of the method. The results lead to the astrophysical factor $S_{17}(0)=$ $18.7 \pm 1.9 \mathrm{eV} \cdot \mathrm{b}$ for the key reaction for solar neutrino production ${ }^{7} \mathrm{Be}(\mathrm{p}, \gamma){ }^{8} \mathrm{~B}$. It is consistent with the values from other indirect methods and most direct measurements, but one. Breakup reactions can be measured with radioactive beams as weak as a few particles per second, and therefore can be used for cases where no direct measurements or other indirect methods for nuclear astrophysics can be applied. We discuss a proposed use of the breakup of the proton drip line nucleus ${ }^{23} \mathrm{Al}$ to obtain spectroscopic information and the stellar reaction rate for ${ }^{22} \mathrm{Mg}(\mathrm{p}, \gamma)^{23} \mathrm{Al}$.
\end{abstract}

PACS. 25.60.-t Reactions induced by unstable nuclei - 25.60.Gc Breakup and momentum distributions 26.65.+t Solar neutrinos - 26.30.+k Nucleosynthesis in novae, supernovae, and other explosive environments

\section{Introduction}

Radiative proton capture reactions are important in nuclear astrophysics, and a large number of reaction chains were found to be needed in nucleosynthesis calculations for static or explosive hydrogen burning scenarios (see e.g. $[1,2])$. This means that more data involving proton capture on unstable nuclei are necessary. In some cases direct experiments are possible, but in many more they are impossible with the present techniques and even with those of the foreseeable future. We have to rely on indirect methods instead. In this presentation we discuss such an indirect method, and we shall concentrate on three particular cases, first to demonstrate the feasibility, then to show the strengths of the method and its limits.

Part of the results discussed were published before, when we originally proposed to extract astrophysical $S$ factors from one-nucleon-removal (or breakup) reactions of loosely bound nuclei at intermediate energies or later [3, $4,5]$. In the present paper, first we use the well studied case of ${ }^{8} \mathrm{~B}$ breakup as a benchmark to demonstrate the usefulness of the method and show the possibili-

\footnotetext{
a e-mail: livius_trache@tamu.edu
}

ties of the Glauber reaction model used. We show that existing experimental data at energies between 30 and $1000 \mathrm{MeV} /$ nucleon $[6,7,8,9,10]$ on a range of light and heavy targets translate into consistent values of the ANC, which is then used to determine the astrophysical factor $S_{17}$ (which gives the reaction rate for the ${ }^{7} \mathrm{Be}(\mathrm{p}, \gamma){ }^{8} \mathrm{~B}$ reaction of crucial importance for the solar neutrino question). We show that the precision of the method is limited to about $10 \%$ by our ability to compute absolute cross sections. Second, we use the same technique for ${ }^{9} \mathrm{C}$ breakup data at $285 \mathrm{MeV} /$ nucleon [7] to determine $S_{18}$ (which gives the rate for the ${ }^{8} \mathrm{~B}(\mathrm{p}, \gamma){ }^{9} \mathrm{C}$ reaction of importance for explosive hydrogen burning) with reasonable accuracy. For a third case, a proposed experiment for the breakup of ${ }^{23} \mathrm{Al}$ is discussed to show that the method is particularly well adapted to rare isotope beams produced using fragmentation. Spectroscopic information is sought in this case. In particular we seek to determine the spin and parity of the ground state of the dripline nucleus ${ }^{23} \mathrm{Al}$ and the $\mathrm{ANC}$, which will be then used to calculate the reaction rates for ${ }^{22} \mathrm{Mg}(\mathrm{p}, \gamma){ }^{23} \mathrm{Al}$. The last part of the present paper will concentrate on this latter case, which has not been discussed before. 


\section{The reaction model}

The method is based on data showing that the structure of halo nuclei is dominated by one or two nucleons orbiting a core $[11,12]$. Consequently, we use the fact that the breakup of halo or loosely bound nuclei is essentially a peripheral process, and therefore, the breakup cross-sections can give information about the wave function of the last nucleon at large distances from the core. More precisely, asymptotic normalization coefficients (ANCs) can be determined. We show that there exists a favorable kinematical window in which breakup reactions are highly peripheral and are dominated by the external part of the wave function and, therefore, the ANC is the better quantity to be extracted. The approach offers an alternative and complementary technique to extracting ANCs from transfer reactions [13].

In the breakup of loosely bound nuclei at intermediate energies, a nucleus $B=(A p)$, where $B$ is a bound state of the core $A$ and the nucleon $p$, is produced by fragmentation from a primary beam, separated and then used to bombard a secondary target. In measurements, the core $A$ is detected, measuring its parallel and transverse momenta and eventually the gamma-rays emitted from its de-excitation. Spectroscopic information can be extracted from these experiments, such as the orbital momentum of the relative motion of the nucleon and the contribution of different core states, typically comparing the measured momentum distributions with those calculated with Glauber models. The integrated cross sections can be used to extract absolute spectroscopic factors [12] or the ANC [3]. The latter approach has the advantage that it is independent of the geometry of the proton binding potential. We note that the ANC $C_{A p}^{B}$ for the nuclear system $A+p \leftrightarrow B$ specifies the amplitude of the tail of the overlap function of the bound state $B$ in the two-body channel $(A p)$ (see, for example [13] and references therein). Fortunately, this ANC is all we need to determine the astrophysical $S$-factor for the radiative proton capture reaction $A(p, \gamma) B$ which is a highly peripheral process. Details about the reaction model are published elsewhere $[5,14]$.

\section{Three particular cases}

\subsection{Breakup of ${ }^{8} \mathrm{~B}$ to determine the $S_{17}$ astrophysical factor}

The calculations presented in [3] have been extended and refined. The Coulomb part of the dissociation cross section was refined by including the final state interaction into calculations and new data on the breakup of ${ }^{8} \mathrm{~B}$ are analyzed $[8,9,10]$. Also a new set of calculations for the breakup of ${ }^{8} \mathrm{~B}$ were made using five sets of different effective $\mathrm{NN}$ interactions. We describe the breakup of ${ }^{8} \mathrm{~B}$ (and in the next subsection of ${ }^{9} \mathrm{C}$ ) in terms of an extended Glauber model. The loosely bound ${ }^{8} \mathrm{~B}\left({ }^{9} \mathrm{C}\right)$ nucleus is moving on a straight line trajectory and the proton and the ${ }^{7} \mathrm{Be}\left({ }^{8} \mathrm{~B}\right)$ core making it, interact independently with the target. The breakup cross sections depend on the protontarget and core-target interactions and on the relative $p$ core motion. The wave function of the ground state of ${ }^{8} \mathrm{~B}$ $\left({ }^{9} \mathrm{C}\right)$ is a mixture of $1 p_{3 / 2}$ and $1 p_{1 / 2}$ orbitals, around a ${ }^{7} \mathrm{Be}\left({ }^{8} \mathrm{~B}\right)$ core. The total ANC $C_{\text {tot }}^{2}=C_{p_{3 / 2}}^{2}+C_{p_{1 / 2}}^{2}$ can be extracted from the measured breakup cross sections.

The calculations reproduce well all the measured parallel and transverse momentum distributions measured so far, on light or heavy targets, giving us confidence in the Glauber model used. We show that the reaction is peripheral in various degrees, depending on the energy and target used. The ${ }^{8} \mathrm{~B}$ ANC is extracted from existing breakup data at energies between $30-1000 \mathrm{MeV} /$ nucleon and on different targets ranging from $\mathrm{C}$ to $\mathrm{Pb}[6,7,8,9,10]$. Two approaches were used. The first is a potential approach. To obtain the folded potentials needed in the $S$-matrix calculations we used the JLM effective nucleon-nucleon interaction [15], using the procedure and the renormalizations of ref. [16]. We applied this technique for energies below $285 \mathrm{MeV} /$ nucleon only and on all targets. In a second approach, the Glauber model in the optical limit was used. The breakup process is treated as multiple elementary interactions between partners' nucleons, and the cross sections and the complex scattering amplitudes are taken from the literature. Calculations were done using different ranges for the elementary interactions: zero range, $1.5 \mathrm{fm}$ ("standard"), $2.5 \mathrm{fm}$ and individual ranges for each $\mathrm{NN}$ component ("Ray") [17]. No new parameters were adjusted. The contribution of the ${ }^{7}$ Be core excitation was calculated for each target and at each energy using the data from an experiment which disentangle it [10], and corrected for in all cases. For details on the procedure see [5]. In fig. 1 we show that from the widely varying breakup cross sections (panel a)) on all targets and at so different energies, we extract ANCs which are consistent with a constant value (panel b)). However, we see that a certain dependence on the NN interaction used exists, which points to the limitations of our present knowledge of the effective nucleon-nucleon interactions.

If we take the unweighted average of all 31 determinations we find an ANC $C_{t o t}^{2}(J L M)=0.483 \pm 0.050 \mathrm{fm}^{-1}$ (fig. 1). The value is in agreement with that determined using the $\left({ }^{7} \mathrm{Be},{ }^{8} \mathrm{~B}\right)$ proton transfer reactions at $12 \mathrm{MeV} / u[18,19]$. The two values agree well, in spite of the differences in the energy ranges and in the reaction mechanisms involved. The ANC extracted leads to the astrophysical factor $S_{17}(0)=18.7 \pm 1.9 \mathrm{eV} \cdot \mathrm{b}$ for the key reaction for solar neutrino production ${ }^{7} \mathrm{Be}(\mathrm{p}, \gamma)^{8} \mathrm{~B}$. The uncertainties quoted are only the standard deviation of the individual values around the average, involving therefore the experimental and theoretical uncertainties. This $10 \%$ error bar is probably a good measure of the precision we can claim from the method at this point in time, due essentially to the uncertainties in the cross section calculations. The $S_{17}(0)$ value we extract is also in agreement with those extracted from indirect methods and with most of the direct determinations (see the discussions in $[20,21$, $22]$ ), but one which stands out in its claim of a larger value and very small error [23]. There are currently many 

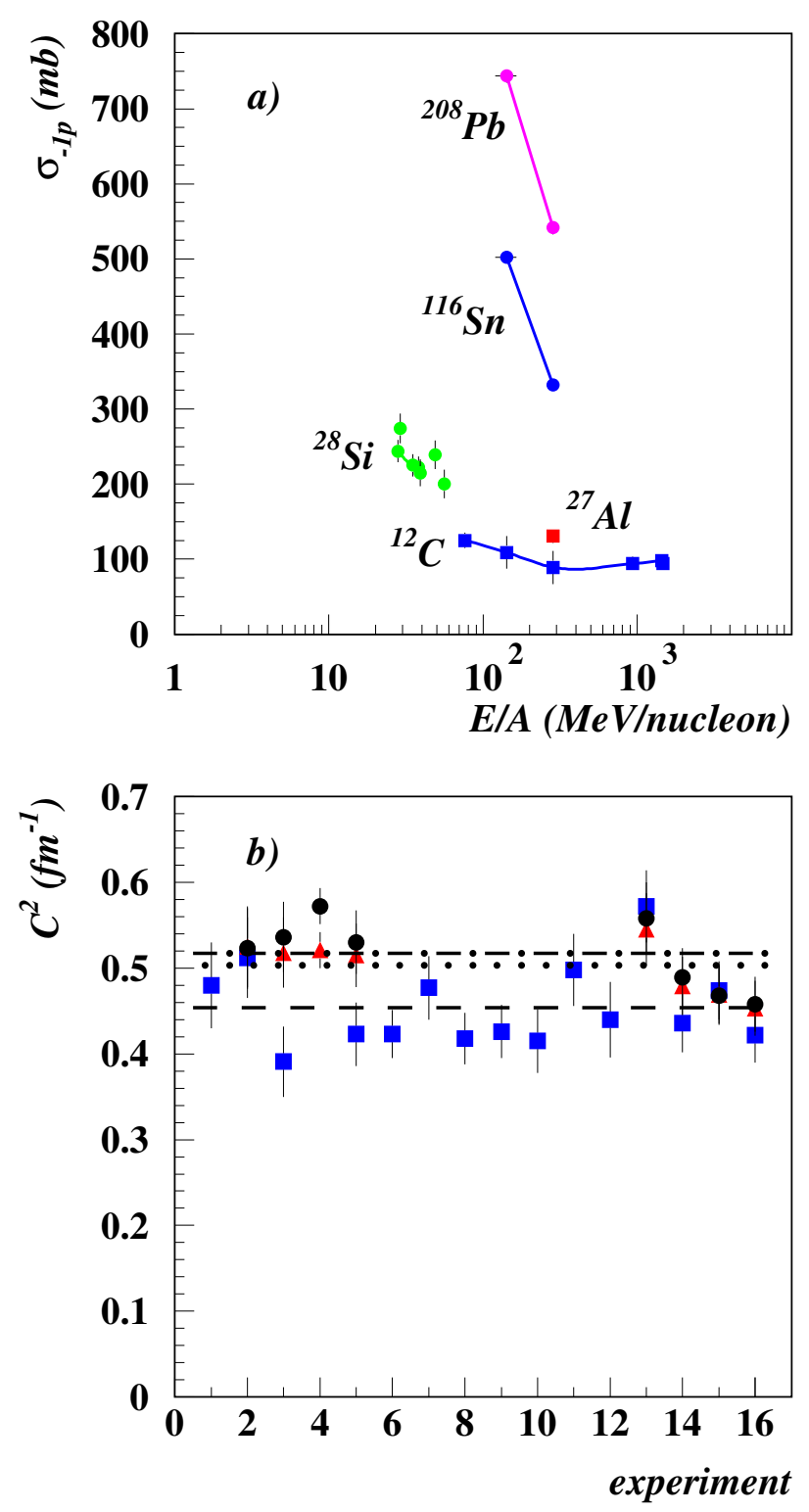

Fig. 1. a) The one-proton-removal cross sections on $\mathrm{C}, \mathrm{Al}, \mathrm{Sn}$ and $\mathrm{Pb}$ targets, depending on energy. b) The ANCs determined from the breakup of ${ }^{8} \mathrm{~B}$ at $28-1000 \mathrm{MeV} /$ nucleon using the data above and various effective interactions: JLM (squares), "standard" (circles) and "Ray" (triangles). The dashed, dotted and dash-dotted lines are the averages of the three interactions above, in that order. List of experiments in ref. [5].

evaluations of existing or new data and variations occur in the central values and uncertainties of the determinations. It is difficult to quote all of them and is not our intention to do so here. However, we notice that our average value of $S_{17}(0)$ is very close to the "low" values obtained from Coulomb dissociation data and some direct data $S_{17}(0)=$ $18.6 \pm 0.4(\exp ) \pm 1.1$ (syst) $\mathrm{eV} \cdot \mathrm{b}[21]$. It is also in reasonable agreement with the average value obtained by Cyburt et al. [24] $S_{17}(0)=20.8 \pm 0.6$ (stat) \pm 1.0 (syst) eV · b using all radiative capture data in the assumption they are completely independent. The difference between our value and the value obtained from the direct measurement of Junghans et al. [23] $S_{17}(0)=22.1 \pm 0.6$ (stat) \pm 0.6 (theor) $\mathrm{eV} \cdot \mathrm{b}$ still exists and is only relevant if the small uncertainty of the latter is true, given the fact that it involves extrapolation. It would, of course, be interesting to understand why the results differ. The difficulties encountered by the direct methods, both experimental (very small cross sections, difficult targets, etc...) and theoretical (extrapolations), are known (see, e.g., ref. [25]). One important factor in any indirect determination of the astrophysical $S$-factor is that of the accuracy of the theoretical calculations involved. Much effort is done currently, e.g., to investigate the accuracy of the absolute values of the calculations used in the analysis of the Coulomb dissociation experiments [26, 27]. We did our part above, using different NN interactions. Our central value is about $1 \sigma$ lower than the average central value obtained by Cyburt et al. [24] in a recent analysis that uses all of the best available capture data, under the assumption that they are independent. Including the uncertainty quoted by Cyburt et al. our results are consistent at the $1 \sigma$ level.

\subsection{Breakup of ${ }^{9} \mathrm{C}$ to determine $\mathrm{S}_{18}$}

The same procedures have been applied for ${ }^{9} \mathrm{C}$ to determine the astrophysical $S_{18}$ factor for the reaction ${ }^{8} \mathrm{~B}(p, \gamma){ }^{9} \mathrm{C}$. The reaction is important in hot $p p$-chains as it can provide a starting point for an alternative path across the $A=8$ mass gap [2]. The ANC for ${ }^{9} \mathrm{C} \rightarrow{ }^{8} \mathrm{~B}+p$, has been determined using existing experimental data for the breakup of ${ }^{9} \mathrm{C}$ projectiles at $285 \mathrm{MeV} / u$ on four different targets: $\mathrm{C}, \mathrm{Al}, \mathrm{Sn}$ and $\mathrm{Pb}$ [7]. No experimental data are available here for momentum distributions. The introduction of the final state interaction in the Coulomb dissociation part does not change the result by much, compared with our previous analysis [4]. We find now $C_{p_{3 / 2}}^{2}+C_{p_{1 / 2}}^{2}=1.26 \pm 0.13 \mathrm{fm}^{-1}$. To calculate the astrophysical $S$-factor we use the potential model. We find $S_{18}(0)=47 \pm 6 \mathrm{eV} \cdot \mathrm{b}$. A very weak dependence on energy is observed: $S(E)=47.3-15.1 E+7.34 E^{2}(E$ in $\mathrm{MeV})$. This result is in very good agreement with other determinations $[28,8]$, but not with one from Coulomb dissociation [29], a fact that we do not understand. We underline that for this case the precision achieved from this determination is the best so far and is sufficient for astrophysical purposes.

\subsection{Breakup of ${ }^{23} \mathrm{Al}$ and the consequences on the ${ }^{22} \mathrm{Mg}(\mathrm{p}, \gamma){ }^{23} \mathrm{Al}$ stellar reaction rate}

Space-based gamma-ray telescopes have the ability to detect $\gamma$-rays of cosmic origin. They already provided strong and direct evidence that nucleosynthesis is an ongoing process through the detection of transitions in the decay of ${ }^{26} \mathrm{Al},{ }^{56} \mathrm{Ni},{ }^{44} \mathrm{Ti}$, etc. Among the expected $\gamma$-ray emitters is ${ }^{22} \mathrm{Na}\left(T_{1 / 2}=2.6 \mathrm{y}\right)$ produced in the thermonuclear runaway and the high-temperature phase in the so-called ONe novae (oxygen-neon novae) through the 


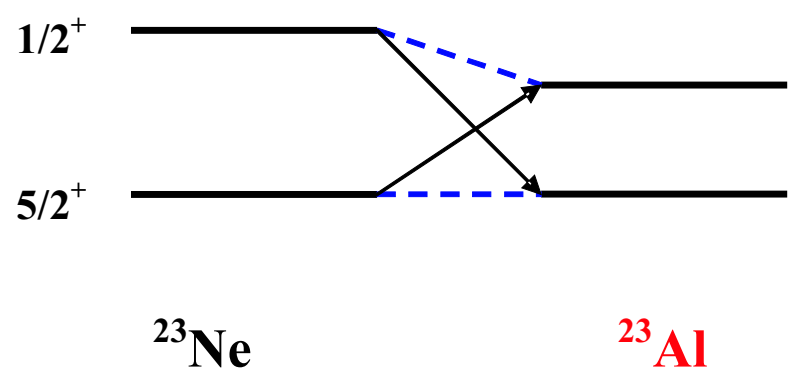

Fig. 2. The level inversion in ${ }^{23} \mathrm{Al}$ suggested in refs. [37,38].

reaction chain ${ }^{20} \mathrm{Ne}(\mathrm{p}, \gamma){ }^{21} \mathrm{Na}(\mathrm{p}, \gamma){ }^{22} \mathrm{Mg}(\beta, \gamma){ }^{22} \mathrm{Na}(\mathrm{NeNa}$ cycle) $[30,31,32]$. Measurements, however, have not detected the $1.275 \mathrm{MeV}$ gamma-ray following the decay of ${ }^{22} \mathrm{Na}$ and have only been able to set an upper limit on its production, a limit which is below the theoretical predictions (see, for example, $[33,34]$ and references therein). This discrepancy may arise from a poor knowledge of the reaction cross sections employed in the network calculations for the rp-process. In particular, it was proposed that the precursor ${ }^{22} \mathrm{Mg}$ can be depleted by the radiative proton capture reaction ${ }^{22} \mathrm{Mg}(\mathrm{p}, \gamma){ }^{23} \mathrm{Al}$ [35], which can result in a serious reduction of the ${ }^{22} \mathrm{Na}$ abundance. The reaction is dominated by direct capture and resonant capture through the first excited state in ${ }^{23} \mathrm{Al}$. There is no direct measurement of the cross section at stellar energies because it is impossible to make a ${ }^{22} \mathrm{Mg}\left(T_{1 / 2}=3.86 \mathrm{~s}\right)$ target and difficult to obtain an intense ${ }^{22} \mathrm{Mg}$ beam. Therefore, currently the rate of this reaction is estimated based on the mass and resonance energy determined experimentally [36] and assuming that the spins and parities are as in the mirror system ${ }^{23} \mathrm{Ne}$.

The nucleus ${ }^{23} \mathrm{Al}$ is a weakly bound proton-rich nucleus $\left(S_{p}=0.123(19) \mathrm{MeV}\right)$ close to the drip line. Recent measurements of the reaction cross sections for $N=10$ isotones and $Z=13$ isotopes around $30 \mathrm{MeV} /$ nucleon on a ${ }^{12} \mathrm{C}$ target found a remarkable enhancement for ${ }^{23} \mathrm{Al}$, which led the authors to the conclusion that it is one of the rare proton halo nuclei [37]. This is explained with a presumed level inversion between the $2 s_{1 / 2}$ and $1 d_{5 / 2}$ orbitals (fig. 2). The inversion was further supported by several microscopic nuclear structure calculations that find $J^{\pi}=1 / 2^{+}$for the ${ }^{23} \mathrm{Al}$ ground state [38]. If the abovementioned inversion is correct, it will affect the radiative capture cross section much more strongly than any other uncertainties. Indeed, assuming such an inversion, we recalculate the astrophysical $S$-factor (fig. 3a) and the stellar reaction rate (fig. $3 \mathrm{~b}$ ) for the ${ }^{22} \mathrm{Mg}(\mathrm{p}, \gamma)^{23} \mathrm{Al}$ reaction and find an increase of 30 to 50 times over the current estimate of the rate for the temperature range $T_{9}=0.1-0.3$. Clearly then, it is important to determine the spin and parity of the low-lying levels in ${ }^{23} \mathrm{Al}$. It is important for both nuclear structure and for its consequences for nuclear astrophysics. As a further complication, the NNDC data base gives $J^{\pi}=3 / 2^{+}$for the ground state of ${ }^{23} \mathrm{Al}$ [39].
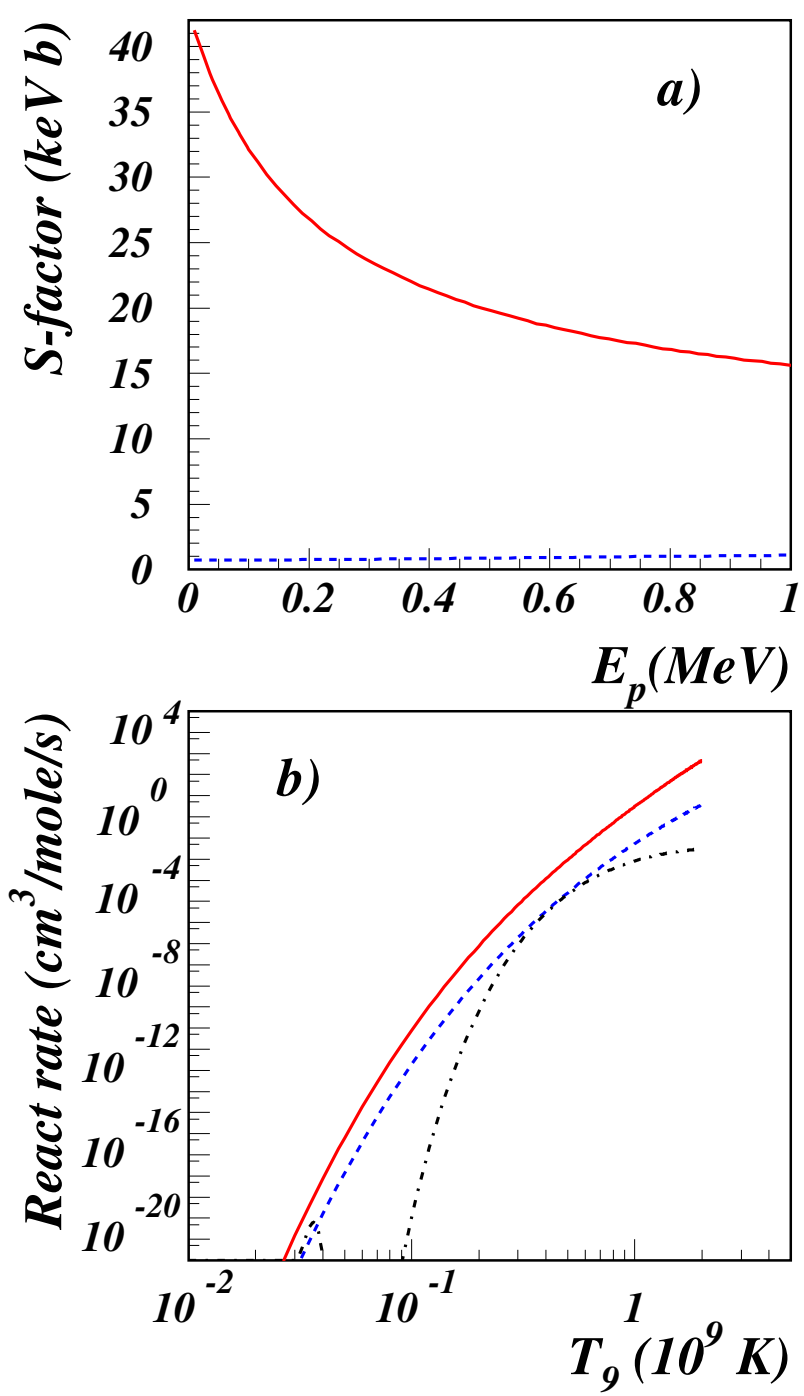

Fig. 3. a) The astrophysical $S$-factor for the ${ }^{22} \mathrm{Mg}(\mathrm{p}, \gamma){ }^{23} \mathrm{Al}$ reaction, calculated assuming $J^{\pi}=5 / 2^{+}$(dashed line), or $J^{\pi}=1 / 2^{+}$(orbital inversion, full line) for the g.s. of ${ }^{23} \mathrm{Al}$. b) The corresponding reaction rate calculated for the case of non-inversion (dashed), or inversion (full line). The dashdotted line shows the resonant contribution of the 1st excited state in ${ }^{23} \mathrm{Al}$.

We proposed the use of intermediate-energy oneproton removal reactions on a light target as a means to determine the structure of the ${ }^{23} \mathrm{Al}$ ground state. Such reactions have proven to be a reliable spectroscopic tool, with advantages in particular for the case of weakly bound isotopes, close to the drip lines $[12,14]$. We calculate that for ${ }^{12} \mathrm{C}\left({ }^{23} \mathrm{Al},{ }^{22} \mathrm{Mg}\right)$ at $60 \mathrm{MeV} /$ nucleon, the parallel momentum distribution is some 2 times narrower for a $2 s_{1 / 2}$ orbital than for a $1 d_{5 / 2}$ orbital (fig. 4) and the associated cross section is about a factor two larger. We intend to compare the calculated momentum distributions and cross sections with the experimental ones and determine the spin and parity of the ${ }^{23} \mathrm{Al}$ ground state. We shall derive the related ANCs and from them, the astrophysical $S$ factor. Calculations for the momentum distributions have 


\section{${ }^{12} \mathrm{C}\left({ }^{23} \mathrm{Al},{ }^{22} \mathrm{Mg}\right)$}
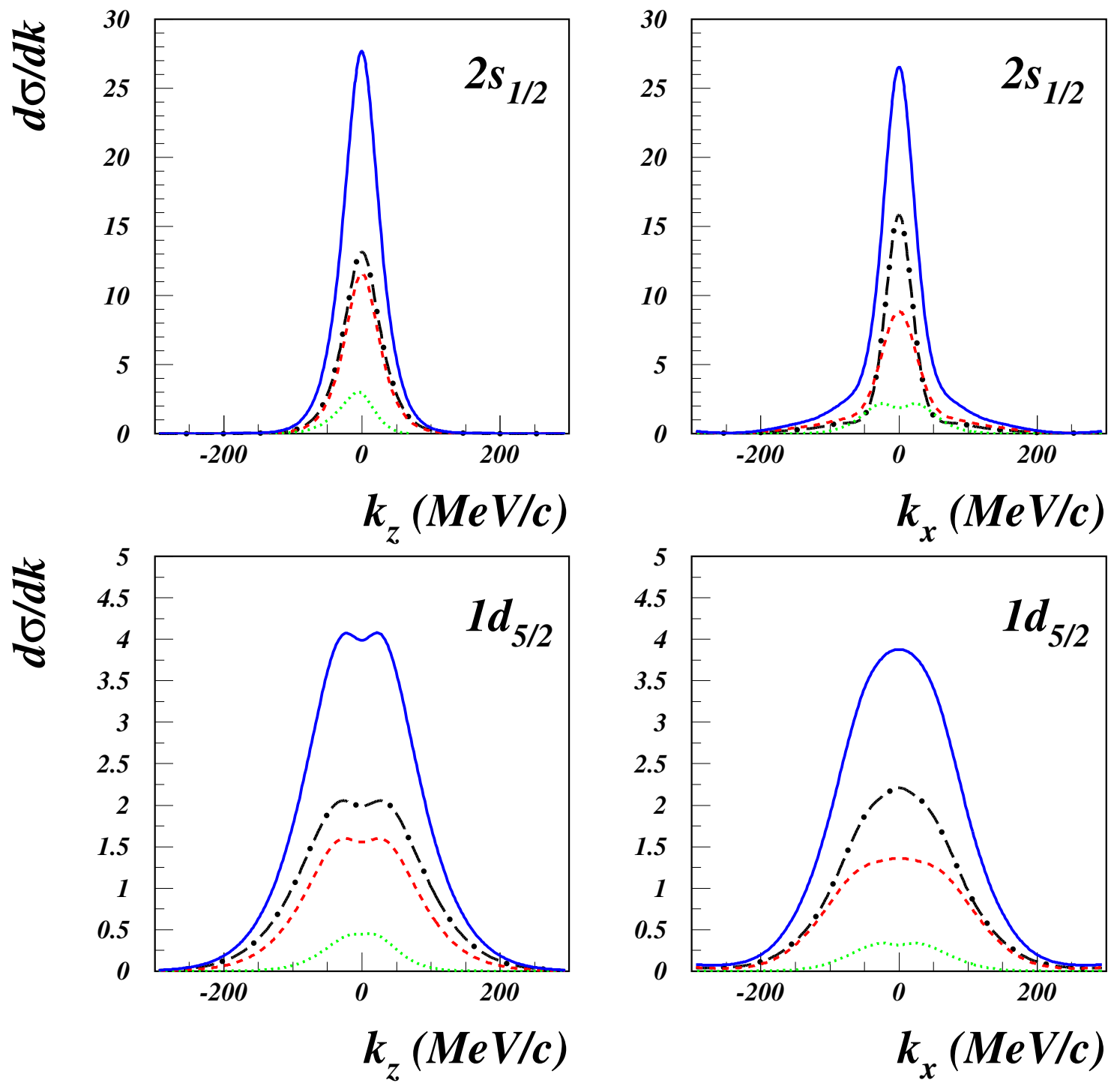

Fig. 4. The calculated parallel (left panels) and transverse (right panels) momentum distributions for the proton-removal (breakup) of $60 \mathrm{MeV} /$ nucleon ${ }^{23} \mathrm{Al}$ on a light target in the two spin assumptions. The curves show the different contributions: stripping (dash-dotted), diffraction dissociation (dashed) and Coulomb (dotted). The full lines are the sum of all contributions. They were all calculated assuming pure $1 d_{5 / 2}$ or $2 s_{1 / 2}$ orbitals, respectively.

been performed with procedures similar to those used previously $[3,14]$. The first step is the calculation of the singleparticle density in ${ }^{22} \mathrm{Mg}$ using a spherical HF+BCS calculation with the density energy functional of Beiner and Lombard [40]. The experimental proton separation energy in ${ }^{23} \mathrm{Al}, S_{p}=0.123 \mathrm{MeV}$, was reproduced. There are two possibilities for the spin-parity of the ground state: $J^{\pi}=$ $5 / 2^{+}$or $1 / 2^{+}$. Glauber model calculations have therefore been performed for each case, assuming pure $1 d_{5 / 2}$, or $2 s_{1 / 2}$ orbitals, in order to demonstrate the sensitivity of the technique. Scattering functions defining the stripping and diffraction transition operators were generated with double-folding potentials using the JLM effective interac- tion, renormalized as above. Calculations were done for breakup on a ${ }^{12} \mathrm{C}$ target to minimize the Coulomb effects.

We draw two conclusions from the calculations:

1) The two possible assignments may be resolved on the basis of the inclusive cross sections and momentum distributions. For example the cross section drops by a factor of two if a $1 d_{5 / 2}$ state is assumed rather than $2 s_{1 / 2}$. This is easy to understand because the low-binding energy and the lack of a centrifugal barrier in the case of a $2 s_{1 / 2}$ orbital leads to a much longer tail of the radial wave function than for the case of the $1 d_{5 / 2}$ orbital. Moreover, the very peripheral character of single-nucleon removal reactions means that it is the asymptotic part of the wave 
function that dictates the cross section and momentum distribution. In the case of the latter, the widths of the momentum distributions differ by a factor of two for both the parallel and transverse momenta, reflecting the different behavior of the tails of the wave functions. Cross sections of $97 \mathrm{mb}\left(2 s_{1 / 2}\right)$, and of $42 \mathrm{mb}\left(1 d_{5 / 2}\right)$ were found for the two ground-state spin-parity assignments. The corresponding widths (FWHM) of the distributions are predicted to be $60 \mathrm{MeV} / c$, and $180 \mathrm{MeV} / c$, respectively.

2) The shape of momentum distributions is extremely selective - narrow for a $2 s_{1 / 2}$ state and broad with a flat top and a small central dip for removal of a $1 d_{5 / 2}$ (fig. 4 ).

A study of one-proton removal from ${ }^{23} \mathrm{Al}$ should, therefore, allow the spin-parity of the ground state of ${ }^{23} \mathrm{Al}$ to be deduced. Measurements of the cross sections and momentum distributions in coincidence with gamma-rays from the ${ }^{22} \mathrm{Mg}$ core will allow us to disentangle the detailed structure of the wave function, and in particular to deduce the spectroscopic factors for the various configurations. This spectroscopic information will also be valuable to determine if ${ }^{23} \mathrm{Al}$ is deformed or spherical. Currently experimental studies of Coulomb dissociation of ${ }^{23} \mathrm{Al}$ are carried out at RIKEN to determine the gammaray width of its first excited state. The results in ref. [41] $\Gamma_{\gamma}=7.2 \pm 1.4 \cdot 10^{-7} \mathrm{eV}$ agree with the value estimated by us using single-particle wave functions for the $E 2$ transition: $\Gamma_{\gamma}=6 \cdot 10^{-7} \mathrm{eV}$ and used in the present calculation of the resonant term (shown in fig. $3 \mathrm{~b}$ ) for the capture reaction rate ${ }^{22} \mathrm{Mg}(p, \gamma)^{23} \mathrm{Al}$.

\section{Conclusions}

In conclusion, we have shown that one-proton-removal reactions at intermediate energies can be used to obtain astrophysical $S$-factors at stellar energies for radiative proton capture reactions. Difficult or impossible direct measurements for nuclear astrophysics at very low energies can be replaced by indirect measurements with radioactive beams at larger energies. We find that a kinematic window exists at $30-150 \mathrm{MeV} /$ nucleon where the reactions are peripheral and the relevant ANC can be determined. The method is particularly useful because it can be used for rare isotopes, for poor-quality radioactive beams obtained from fragmentation, with cocktail beams and with low intensity beams. It was shown that breakup at intermediate energies can be studied with beams as low as a few particles/s [42]. Our results from the use of different NN interactions remind us of the fact that the precision of all indirect methods depends not only on the precision of the experiments but also on the accuracy of the calculations. Our findings may give a measure of the present status of accuracy.

This work was supported in part by the U.S. Department of Energy under Grant No. DE-FG03-93ER40773, by the Romanian Ministry for Research and Education under contract No. 555/2000, and by the Robert A. Welch Foundation.

\section{References}

1. J.N. Bahcall, M.H. Pinsoneault, S. Basu, Astrophys. J. 555, 990 (2001).

2. M. Wiescher et al., Astrophys. J. 343, 352 (1989).

3. L. Trache, F. Carstoiu, C.A. Gagliardi, R.E. Tribble, Phys. Rev. Lett. 87, 271102 (2001).

4. L. Trache et al., Phys. Rev. C 66, 035801 (2002).

5. L. Trache, F. Carstoiu, C.A. Gagliardi, R.E. Tribble, Phys. Rev. C 69, 032802(R) (2004).

6. F. Negoita et al., Phys. Rev. C 54, 1787 (1996).

7. B. Blank et al., Nucl. Phys. A 624, 242 (1997).

8. J. Enders et al., Phys. Rev. C 67, 064301 (2003).

9. R. Warner et al., to be published in Phys. Rev. C.

10. D. Cortina-Gil et al., Nucl. Phys. A 720,3 (2003).

11. I. Tanihata, J. Phys. G 22, 157 (1996).

12. P.G. Hansen, B.M. Sherrill, Nucl. Phys. A 693, 133 (2001).

13. A.M. Mukhamedzhanov et al., Phys. Rev. C 63, 024612 (2001).

14. E. Sauvan et al., Phys. Lett. B 491, 1 (2000); Phys. Rev. C 69, 044603 (2004).

15. J.P. Jeukenne, A. Lejeune, C. Mahaux, Phys. Rev. C 16, 80 (1977).

16. L. Trache et al., Phys. Rev. C 61, 024612 (2000).

17. L. Ray, Phys. Rev. C 20, 1857 (1979).

18. A. Azhari et al., Phys. Rev. Lett. 82, 3960 (1999).

19. G. Tabacaru et al., Phys. Rev. C 73, 025808 (2006).

20. F. Schumann et al., Phys. Rev. Lett. 90, 232501 (2003) and references therein.

21. B. Davids, S. Typel, Phys. Rev. C 68, 045802 (2003).

22. F. Hammache et al., Nucl. Phys. A 746, 370c (2004).

23. A.R. Junghans et al., Phys. Rev. C 68, 065803 (2003).

24. R.H. Cyburt, B. Davids, B.K. Jennings, Phys. Rev. C 70, 045501 (2004).

25. F. Strieder et al., Eur. Phys. J. A 3, 1 (1998).

26. H. Esbensen, G.F. Bertsch, K.A. Snover, Phys. Rev. Lett. 94, 042502 (2005).

27. C. Bertulani, Phys. Rev. Lett. 94, 072701 (2005).

28. D. Beaumel et al., Phys. Lett. B 514, 226 (2001).

29. T. Motobayashi, Nucl. Phys. A 718, 101c (2002); RIKEN Accelerator Progress Report 1999, 33, 64 (2000) and 2002, 36, 64 (2003).

30. S. Starrfield, J.W. Truran, M. Wiescher, W.M. Sparks, Mon. Not. R. Astron. Soc. 296, 502 (1998).

31. J. Jose, A. Coc, M. Hernanz, Astrophys. J. 520, 347 (1999).

32. S. Wanajo, M. Hashimoto, K. Homono, Astrophys. J. 523, 409 (1999)

33. I. Iyundin et al., Astron. Astrophys. 300, 422 (1995).

34. R. Diehl, Nucl. Phys. A 718, 52c (2003) and references therein.

35. M. Wiescher et al., Nucl. Phys. A 484, 90 (1988).

36. J. Caggiano et al., Phys. Rev. C 64, 025802 (2001).

37. X.Z. Cai et al., Phys. Rev. C 65, 024610 (2002).

38. H.-Y. Zhang et al., Chin. Phys. Lett. 19, 1599 (2002); 20, 46; 1234 (2003).

39. National Nuclear Data Center, BNL. http://www.nndc. bnl.gov.

40. M. Beiner, R.J. Lombard, Ann. Phys. (N.Y.) 86, 262 (1974).

41. T. Gomi et al., Nucl. Phys. A 718, 508c (2003); 734, Suppl. 1, E77 (2004); 758, 761c (2005).

42. V. Maddalena et al., Phys. Rev. C 63, 024613 (2001). 